\title{
Correlation between microdilution, Etest, and disk diffusion methods for antifungal susceptibility testing of fluconazole against Candida sp. blood isolates
}

\author{
Everardo Albuquerque Menezes ${ }^{[1]}$, Antônio Alexandre de Vasconcelos Júnior ${ }^{[1]}$, \\ Maria Rozzelê Ferreira Ângelo ${ }^{[2]}$, Maria da Conceição dos Santos Oliveira Cunha ${ }^{[1]}$ \\ and Francisco Afrânio Cunha ${ }^{[1]}$
}

[1]. Laboratório de Pesquisa em Microbiologia de Leveduras, Departamento de Análises Clínicas e Toxicológicas, Faculdade de Farmácia, Universidade Federal do Ceará, Fortaleza, CE. [2]. Hospital de Fortaleza, Fortaleza, CE.

\begin{abstract}
Introduction: Antifungal susceptibility testing assists in finding the appropriate treatment for fungal infections, which are increasingly common. However, such testing is not very widespread. There are several existing methods, and the correlation between such methods was evaluated in this study. Methods: The susceptibility to fluconazole of 35 strains of Candida sp. isolated from blood cultures was evaluated by the following methods: microdilution, Etest, and disk diffusion. Results: The correlation between the methods was around $90 \%$. Conclusions: The disk diffusion test exhibited a good correlation and can be used in laboratory routines to detect strains of Candida sp. that are resistant to fluconazole.
\end{abstract}

Keywords: Disk diffusion. Antifungal susceptibility. Fluconazole.

Antifungal susceptibility testing is a notable breakthrough in the treatment of fungal infections and is the primary tool in determining the appropriate antifungal therapy ${ }^{1}$. Several methods are available to evaluate the antifungal susceptibility of Candida sp., and the main goal of these methods is to detect resistance in vitro. The most common methods are: broth microdilution (BMD), disk diffusion (DD), and Etest ${ }^{2-4}$. BMD is a test that can be somewhat troublesome to conduct in a laboratory routine, and Etest is still an expensive method. Therefore, DD is the most appropriate test to be applied in the detection of resistant strains due to its simplicity and ease of execution ${ }^{5}$.

The aims of this study were to assess the susceptibility to fluconazole of Candida sp. strains, isolated from blood cultures of patients in the state of Ceará, using three different methods and to measure the correlation between these methods.

We evaluated 35 isolates of Candida sp. (16 Candida tropicalis and 19 Candida parapsilosis) isolated from blood cultures of ICU (Intensive Care Unit) patients at Hospital Geral de Fortaleza, Ceará, Brazil. The yeasts were isolated on a potato agar and incubated at $35^{\circ} \mathrm{C}$ for $24 / 48 \mathrm{~h}$. The presumptive identification was performed on HiCrome Candida ${ }^{\circledR}$ agar medium (Mumbai, India). The identification was confirmed by a microculture on rice agar with Tween 80 and with the API $20 \mathrm{C}$ AUX® kit (BioMérieux, Marcy-I’Étoile, France) ${ }^{5}$.

Address to: Dr. Antônio Alexandre de Vasconcelos Júnior. Laboratótio de Microbiologia de Leveduras/UFC. R. Capitão Francisco Pedro 1210, Rodolfo Teófilo, 60430-370 Fortaleza, CE, Brasil.

Phone: 5585 3366-8266

e-mail: alexandrevasconcelosjr@gmail.com

Received in 30/03/2011

Accepted in 06/12/2011
Antifungal susceptibility tests were conducted by the methods of broth microdilution in RPMI, Etest, and disk diffusion in accordance with the Clinical Laboratory Standards Institute (CLSI) documents. The break points used in this study were those suggested in protocols M27-A3 and M44-A22-4. The break points for susceptibility interpretation are as follows: for fluconazole, Minimum Inhibitory Concentration (MIC) of $\leq 8 \mu \mathrm{g} / \mathrm{ml}$ for susceptible (S); MICs of 16 to $32 \mu \mathrm{g} / \mathrm{ml}$ for susceptible dose dependent (SDD); and MICs of $\geq 64 \mu \mathrm{g} / \mathrm{ml}$ for resistant (R) for BMD and Etest methods. The interpretive criteria for the fluconazole disk diffusion tests were: (S), zone diameters $\geq 19 \mathrm{~mm}$; $\mathrm{SDD}, 15$ to $18 \mathrm{~mm}$; (R), zone diameters $\leq 14 \mathrm{~mm}^{2,3}$.

A comparison of methodologies was performed by using analysis of the error as recommended by the CLSI. The definitions of error used in this study were as follows: the Very Major Error (VME) that occurs when one strain is detected (R) by the method of BMD (considered standard) and this same strain appears as sensitive when tested by the DD method, the Major Error (ME) that is observed when a strain is shown as (S) by BMD and (R) by $\mathrm{DD}$, and the Minor error (M) that occurs when the result shows BMD as susceptible dose dependent (SDD) and DD is (S) or (R). Essential Agreement (EA) is calculated by using the formula: $\mathrm{EA}=100-\mathrm{VME}+\mathrm{ME}+\mathrm{M}$. In general, the methods showed a good correlation to the VME, which should be $<1.5 \%$, and the agreement between the methodologies should be $>90 \%{ }^{2}$.

Table 1 shows the results obtained in this study: the DD method showed a good agreement with the other methods tested. In studies with Candida sp. isolated in Brazil, a correlation approaching $70 \%$ between the methods was found. In our study, the agreement was higher, exceeding $90 \%{ }^{6,7}$. 
Based on these results and on studies that evaluated a high number of strains of Candida sp., we can conclude that the disk diffusion test correlated well with the other methods of assessing susceptibility. It is inexpensive, easy to perform, and can be implemented in laboratory routines that provide results in $24 \mathrm{~h}^{5,8}$.

TABLE 1 - Correlation between methods for antifungal susceptibility testing of fluconazole against Candida sp.

\begin{tabular}{lccc}
\hline Strains (n) and method & Range $^{\mathbf{a}}$ & $\mathbf{S} / \mathbf{S D D} / \mathbf{R}^{\mathbf{b}}$ & Essential agreement $(\%)^{(\%)}$ \\
\hline Candida parapsilosis (19) & & & - \\
$\quad$ BMD & $0.12-64$ & $16 / 1 / 2$ & 90.0 \\
Etest & $1.0-256$ & $15 / 1 / 3$ & 90.0 \\
DD & $0-37$ & $15 / 1 / 3$ & \\
Candida tropicalis (16) & & & - \\
BMD & $0.12-32$ & $15 / 1 / 0$ & 93.0 \\
Etest & $1.0-2.0$ & $16 / 0 / 0$ & 93.0 \\
DD & $23-38$ & $16 / 0 / 0$ & \\
\hline
\end{tabular}

n: number of strains; S/SDD/R: susceptible, susceptible dose dependent, and resistant, respectively; BMD: broth microdilution; DD: disk diffusion; ${ }^{\text {a }}$ : ranges of BMD, Etest, and DD are given as $\mu \mathrm{g} / \mathrm{ml}, \mu \mathrm{g} / \mathrm{ml}$, and halo $\mathrm{mm}$, respectively; ${ }^{\mathrm{b}}$ : number of isolates.

\section{CONFLICT OF INTEREST}

The authors declare that there is no conflict of interest.

\section{FINANCIAL SUPPORT}

This study was funded by the Conselho Nacional de Desenvolvimento Cientifico e Tecnológico (CNPq).

\section{RESULTS}

1. Chen SCA, Playford G, Sorrell TC. Antifungal therapy in invasive fungal infections. Curr Opin Pharm 2010; 10:522-530.

2. Clinical and laboratory Standards Institute (CLSI). Reference Method for Broth Dilution Antifungal Susceptibility Testing of Yeasts. Approved Standard M27-A3, $3^{\text {rd }}$ ed. Wayne, PA: CLSI; 2008a.

3. Clinical and laboratory Standards Institute (CLSI). Method for Antifungal Disk Diffusion Susceptibility Testing of Yeasts: Approved Standard M44-A2. Wayne, PA: CLSI; 2008b.

4. Espinel-Ingroff A, Pfaller M, Cantón M, Pemán J. Emerging Resistance to Azoles and Echinocandins: Clinical Relevance and Laboratory Detection. Curr Fungal Infect Rep 2010; 4:186-195.

5. Menezes EA, Mendes LG, Cunha FA. Resistência a antifúngicos de Candida tropicalis isoladas no Estado do Ceará. Rev Soc Bras Med Trop 2009; 2: 354-355.

6. Negri M, Henriques M, Svidzinski TIE, Paula CR, Oliveira R. Correlation Between Etest s, Disk Diffusion, and Microdilution Methods for Antifungal Susceptibility Testing of Candida Species From Infection and Colonization. J Clin Lab Anal 2009; 23:324-330.

7. Dota KFD, Freitas AR, Consolaro MEL, Svidzinski TIE. Challenge for Clinical Laboratories: Detection of Antifungal Resistance in Candida Species Causing Vulvovaginal Candidiasis. Lab Med 2011; 42:87-93.

8. Pfaller MA, Diekema DJ, Gibbs DL, Newell VA, Meis JF, Gould IM, et al. and the Global Antifungal Surveillance Study. Results from the ARTEMIS DISK Global Antifungal Surveillance Study, 1997 to 2005: an 8.5 year analysis of susceptibilities of Candida species and other yeast species to ûuconazole and voriconazole determined by CLSI standardized disk diffusion testing. J Clin Microbiol 2007; 45:1735-1745. 